\title{
Serum MASP-1 in complex with MBL activates endothelial cells
}

Márton Megyeri ${ }^{1,5}$, Péter K. Jani ${ }^{2,5}$, Erika Kajdácsi ${ }^{2}$, József Dobó ${ }^{1}$, Endre Schwaner ${ }^{2}$, Balázs Major $^{1}$, János Rigó jr. ${ }^{4}$, Péter Závodszky ${ }^{1}$, Steffen Thiel ${ }^{3}$, László Cervenak ${ }^{2}$ and Péter Gál ${ }^{1,6}$

${ }^{1}$ Institute of Enzymology, Research Centre for Natural Sciences, Hungarian Academy of Sciences, Karolina út 29., H-1113, Budapest, Hungary

${ }^{2} 3^{\text {rd }}$ Department of Medicine, Research Lab, Semmelweis University, Kútvölgyi út 4., H1125 Budapest, Hungary

${ }^{3}$ Department of Biomedicine, Aarhus University, DK-8000 Aarhus C, Denmark

${ }^{4} 1^{\text {st }}$ Department of Obstetrics and Gynecology, Semmelweis University, Baross u. 27., H-1088 Budapest, Hungary

Running title: MBL-MASPs complexes activate endothelial cells

\section{Footnotes:}

${ }^{5}$ Equal contribution

${ }^{6}$ Corresponding author: gal.peter@ttk.mta.hu, Phone: +36-1-2793135, Fax: +36-1-4665465 


\begin{abstract}
The complement system plays an important role in the induction of inflammation. In this study we demonstrate that the initiation complexes of the lectin pathway, consisting of mannose-binding lectin (MBL) and associated serine proteases (MASPs) elicit $\mathrm{Ca}^{2+}$ signaling in cultured endothelial cells (HUVECs). This is in agreement with our previous results showing that the recombinant catalytic fragment of MASP-1 activates endothelial cells by cleaving protease activated receptor 4 . Two other proteases, MASP-2 and MASP-3 are also associated with MBL. Earlier we showed that recombinant catalytic fragment of MASP-2 cannot activate HUVECs, and in this study we demonstrate that the same fragment of MASP3 has also no effect. We find the same to be the case if we use recombinant forms of the Nterminal parts of MASP-1 and MASP-2 which only contain non-enzymatic domains. Moreover, stable zymogen mutant form of MASP-1 was also ineffective to stimulate endothelial cells, which suggests that in vivo MASP-1 have the ability to activate endothelial cells directly as well as to activate the lectin pathway simultaneously. We show that among the components of the MBL-MASPs complexes only MASP-1 is able to trigger response in HUVECs and the proteolytic activity of MASP-1 is essential. Our results strengthen the view that MASP-1 plays a central role in the early innate immune response.
\end{abstract}

Keywords: complement lectin pathway, MBL-associated serine proteases, endothelial cell, cell activation, protease activated receptor

\footnotetext{
Abbreviations: HUVEC: human umbilical vein endothelial cell; MAPK: mitogen-activated protein kinase; MASP: MBL-associated serine protease; MASP-1cf: MASP-1 catalytic fragment; MAp: MBL-associated protein; MBL: mannan-binding lectin; PAR: protease activated receptor
} 


\section{Introduction}

The complement system is a major effector arm of innate immunity. Its main functions are labeling and eliminating invading pathogens and altered self structures, triggering inflammation, and modulating the adaptive immune response (Ricklin et al., 2010). This network of about 40 proteins includes recognition molecules, serine proteases, thioester containing molecules, cell-surface-receptors and regulators. The danger signals presented by invading pathogens (PAMP: pathogen associated molecular pattern) or by altered host cells (DAMP: damage or danger associated molecular pattern) are recognized by pattern recognition molecules. The lectin pathway of the complement system has at least five different pattern recognition molecules: mannose-binding lectin (MBL), H-, L- and M-ficolin (also named ficolin-3, -2, -1, respectively) (Thiel, 2007) and collectin-11 (also named collectin kidney 1) (Hansen et al., 2010). These molecules may recognize specific patterns of carbohydrate arrays on the surface of microorganisms, hence the name "lectin pathway". The recognition event is converted into an enzymatic signal by serine proteases that are associated with the pattern recognition molecules. MBL and ficolins associate with three serine proteases: MASP (MBL-associated serine protease)-1, -2 and -3 (Matsushita and Fujita, 1992; Thiel et al., 1997; Dahl et al., 2001), and with two non-catalytic proteins MAp44 (Degn et al., 2009; Skjoedt et al., 2010) and MAp19 (Stover et al., 1999; Takahashi et al., 1999). The composition and the stoichiometry of the MBL-MASPs and ficolin-MASPs complexes are not known but it seems likely that a number of different complexes exist in the plasma. When the pattern recognition molecules bind to the target structure, the zymogen serine proteases become activated. An Arg-Ile bond is cleaved in the activation loop, which results in conformational change in the serine protease domain. Recently, we have shown that MASP-1, the most abundant protease component of the lectin pathway, controls lectin pathway activation (Degn et al., 2012; Héja et al., 2012a; 2012b; Kocsis et al., 2010; Megyeri et al., 2013). MASP-1 autoactivates and cleaves zymogen MASP-2, which then cleaves C4 and C2 components (Kidmose et al., 2012). The resulting C3 convertase (C4b2a) is responsible for maintenance and amplification of the complement cascade.

One of the most important functions of the complement cascade is to trigger inflammation. The split products released during complement activation (e.g. anaphylatoxins: C3a, C5a) activates leukocytes and endothelial cells through $G$ protein-coupled receptors. Recently, we have discovered a new function of the complement system that may contribute to the pro-inflammatory effect of complement activation. MASP-1, like thrombin, is capable 
of activating endothelial cells directly through cleavage of protease activated receptor 4 (PAR4) (Megyeri et al., 2009).

Endothelial cells, besides their obvious barrier function, have significant role in several important physiological and pathophysiological processes. Among others, endothelial cells coordinate inflammatory response involving a range of receptors and the capability of producing pro-inflammatory cytokines and adhesion molecules. Endothelial cell activation through PARs involves intracellular $\mathrm{Ca}^{2+}$-mobilization, which results in downstream cytoskeleton reorganization, mitogen-activated protein kinase (MAPK) activation and a shift in the pattern of gene expression. Thrombin and other agonist proteases, induce shape and permeability changes in the vascular endothelium and mobilize adhesive molecules to the cell surface promoting leukocyte rolling, adhesion and transendothelial migration. Previously we demonstrated that a recombinant catalytic fragment of MASP-1 (rMASP-1cf), which contains the serine protease (SP) domain and the two complement control protein (CCP) modules, cleaves PAR4 and elicits $\mathrm{Ca}^{2+}$ signaling, NF- $\mathrm{B}$ nuclear translocation and p38 MAPK phosphorylation in human umbilical vein endothelial cells (HUVECs). Although the catalytic fragment is enzymatically equivalent to the full-length-molecule, it cannot bind to MBL or ficolins because the $\mathrm{N}$-terminal three domains are missing. Under physiological conditions MASP-1 is a part of the MBL-MASPs complexes as well as part of ficolin-MASPs complexes. The complex formation between MASP-1 and MBL may change the accessibility of the active site of the protease for external substrates, like PARs. Moreover, it was shown earlier that MBL binds to endothelial cells (Oroszlán et al., 2007), and we cannot exclude the possibility that this binding modulates the effect of MASP-1 on the cells besides changing the local concentration of MASP-1 on the surface of the cells. It is therefore important to examine whether native MASPs in complexes with MBL are able to activate endothelial cells.

In the present study we analyzed the effect of MBL-MASPs complexes on endothelial cells. We isolated MBL-MASPs complexes from human plasma and studied their ability to stimulate endothelial cells. We also wanted to show unambiguously that proteolytically inactive MASP-1 and the N-terminal non-catalytic regions of MASP-1/3 and MASP-2 are unable to activate cells. Moreover, we asked whether zymogen MASP-1 is able to activate endothelial cells similarly to activated MASP-1. Finally, we also examined whether the catalytic activity of the third protease component of the lectin pathway, MASP-3, exerts any effect on HUVECs. 


\section{Materials and Methods}

\subsection{Materials}

CNBr-activated Sepharose 4B was from GE Healthcare. Coupling of mannan to CNBr-activated resin was performed according to the manufacturer's instructions. All the other reagents were purchased from Sigma-Aldrich, unless otherwise stated. Lyophilized plasma $\mathrm{C} 1$ inhibitor (C1-inh, Berinert $\mathrm{P}$ ) was from ZLB Behring, and it was further purified by cation-exchange chromatography (Source 15S column; GE Healthcare) and gel filtration (Sephadex 75 HiLoad 16/60; GE Healthcare).

\subsection{Recombinant MASP fragments}

DNA constructs of recombinant human MASP-1, and -2 catalytic fragments encoding the CCP1-CCP2-SP region (rMASPcf) were used for expression in E. coli as described (Ambrus et al., 2003). rMASP-2cf was prepared according to Gál et al. (Gál et al., 2005). The R448Q and S646A (precursor numbering) mutants of rMASP-1cf were made according to Megyeri et al. (Megyeri et al., 2013). Wild-type (wt) rMASP-1cf was produced as described by Dobó et al. (Dobó et al., 2008, 2009), but in the absence of benzamidine. The two-step purification procedure was performed in a single day in order to minimize degradation.

The R448Q and the S646A variants of rMASP-1cf were prepared the same way as the wt enzyme, however in these cases the procedure yields the proenzymic (one-chain) form (Megyeri et al., 2013). The S646A mutant of rMASP-1cf at $100 \mu \mathrm{g} / \mathrm{mL}$ was cleaved by rMASP-2 catalytic fragment $(2.5 \mu \mathrm{g} / \mathrm{mL})$ by overnight incubation at room temperature to bring the enzyme into the two-chain form. After cleavage, the S646A mutant was further purified by repeating the anion exchange chromatographic step on a Source $30 \mathrm{Q}$ column to remove rMASP-2cf. We used rMASP-2cf to cleave rMASP-1cf S646A since the two can be separated due to their distinct chromatographic behavior. The procedure yielded an enzyme, which was in the active conformation, but due to the absence of the catalytic serine it had no catalytic activity. Since MASP-2 does not activate endothelial cells (Megyeri et al., 2009) traces of MASP-2 (if any) would not interfere with the cell activation assay.

Wild type proenzymic rMASP-3cf was produced as previously described (Megyeri et al., 2013). Purified rMASP-3cf (400 $\mu \mathrm{g} / \mathrm{mL})$ was activated by cleavage with rMASP-1cf (25 $\mu \mathrm{g} / \mathrm{mL}$ ) at $37{ }^{\circ} \mathrm{C}$ for 3 hours. In the next step rMASP-1cf were removed from the samples by cation exchange chromatography on a SP Sepharose High Performance (GE Healthcare) 
column. The N-terminal fragments (CUB1-EGF-CUB2) of MASP-1 and MASP-2 were prepared as described by Paréj et al. (2014).

\subsection{Purification of MBL-MASPs complexes}

MBL-MASPs complexes were purified by a modified method described by Matsushita et al (Matsushita et al., 2000b). Briefly: plasma samples were clotted by the addition $25 \mathrm{mM}$ $\mathrm{CaCl}_{2}$ and filtered through muslin and the resulting serum was kept at $-80^{\circ} \mathrm{C}$ until further usage. Serum samples were then thawed and incubated overnight at $4{ }^{\circ} \mathrm{C}$ with yeast mannanSepharose 4B resin using $10 \mathrm{mM}$ imidazole buffer ( $\mathrm{pH}$ 6.0) containing $0.2 \mathrm{M} \mathrm{NaCl}, 20 \mathrm{mM}$ $\mathrm{CaCl}_{2}, 0.1 \mathrm{mg} / \mathrm{ml}$ Pefabloc, and $2 \%$ mannitol. After washing the resin with the same buffer, the MBL-MASPs complexes were eluted from the mannan-beads using the above buffer containing 0.3 M mannose. The MBL and MASP-1 concentrations in the eluted samples were determined by sandwich-type immune-assays (see below) as previously described (Thiel et al., 2012; 2002).

To increase the yield of the purified MBL-MASPs complexes we modified the protocol by adding $10 \mu \mathrm{g} / \mathrm{ml}$ recombinant MBL (Jensenius et al., 2003) to the serum samples before incubation with mannan-Sepharose 4B resin. After elution, the fractions were checked by TRIFMA and the appropriate fractions were pooled and concentrated on a spin concentrator and dialyzed against Hank's Balanced Salt Solution (HBSS) and kept at $-80^{\circ} \mathrm{C}$ until use on endothelial cells.

\subsection{C1-inhibitor treatment}

In some control experiments the activity of the plasma derived and recombinant MASP-1 was blocked by $\mathrm{C} 1$-inhibitor. The proteases were incubated with $\mathrm{C} 1$-inhibitor in a 1:1 molar ratio at $37^{\circ} \mathrm{C}$ for $20 \mathrm{~min}$ before adding to the HUVECs.

\subsection{Western blot analysis of purified MBL-MASP complexes}

The Western blot analysis of the purified complexes was performed using the antibodies and the protocol described in detail in Degn et al. (Degn et al., 2010). The rabbit antiserum towards MASP-1, Rabbit64, was raised against the 19 amino acid located on the Cterminus of MASP-1, and thus recognizes the serine protease domain of MASP-1, also called the B chain. Images were taken by CCD camera (LAS-3000, Fuji) and analyzed by the software supplied with the camera. 


\subsection{Assay of the lectin pathway components in the purified complexes}

Concentration measurements of MASP-1 (Thiel et al., 2012), MASP-3 and MAp44 (Degn et al., 2010), MASP-2 (Møller-Kristensen et al., 2003), MAp19 (Degn et al., 2011) and MBL (Thiel et al., 2002) in the purified complexes were carried out, as described previously. All assays are based on sandwich-type immunoassays performed in microtiter wells with the use of biotin-labeled detecting Abs followed by europium-labeled streptavidin. The amount of europium bound in the wells is subsequently measured by time-resolved fluorescence, and this assay type is therefore referred to as a time-resolved immunofluorimetric assay (TRIFMA).

\subsection{Preparation and culture of HUVECs and measurement of intracellular $\mathrm{Ca}^{2+}$ signaling by} fluorescence microscopy

The preparation and culture of endothelial cells, and the $\mathrm{Ca}^{2+}$-mobilization measurements were performed as described previously (Megyeri et al., 2009). Briefly, HUVECs were cultured in 96-well plates for 1 day then loaded with Fluo-4-AM $(2 \mu \mathrm{M})$. Three photos were taken on an Olympus XM-10 camera as a base-line then the activator was added to the cells and the response was measured for 2 minutes. The maximum fluorescence intensity and the base-line fluorescence intensity were calculated, and the differences were compared to those of positive controls.

\subsection{Statistical analysis}

Data were compared using one-way ANOVA with Tukey's post-test and Student's $t$ test (GraphPad Prism 4.02 software, GraphPad, http://www.graphpad.com/). A p-value less than 0.05 was considered significant. Data are presented as means $\pm \mathrm{SEM}$.

\section{Results}

\subsection{Purification of MBL-MASPs complexes form human plasma}

To assess whether MBL-MASPs complexes can stimulate endothelial cells, we purified MBL-MASPs complexes from human plasma according to the original protocol of Matsushita et al. (Matsushita et al., 2000a) and measured the quantity of the yielded MBL and MASP-1 in the different fractions (data not shown). We found that MASP-1 co-eluted from the affinity column with MBL and the amount of MBL was proportional to the MASP-1 
concentration. Unfortunately, this protocol was not able to yield sufficient amount of MASP-1 for testing endothelial cell activation. In order to increase the yield of MBL-bound MASP-1, we added recombinant MBL $(10 \mu \mathrm{g} / \mathrm{ml})$ to the serum samples before incubation with the mannan-conjugated Sepharose 4B resin. While the original protocol yielded $8 \mu \mathrm{g}$ MASP-1 from $100 \mathrm{ml}$ serum, the modified one allowed us to isolate $160 \mu \mathrm{g}$ MASP-1 from the same volume of serum. Apparently, the addition of recombinant MBL shifted the equilibrium in such a way that the amount of MBL-MASPs complexes increased considerably at the expense of the ficolin-MASPs complexes. The purified complex was characterized by Western blot analysis after fractionation in reducing conditions and developing the membrane with rabbit antisera (Rabbit 64) towards MASP-1 that recognizes the MASP-1 B chain (Degn et al., 2010). It is known that MASP-1 is readily activated and we also find here that zymogen MASP-1 is autoactivated after the purification and dialysis against the HBSS buffer and subsequent concentration of the samples (Fig. 1).

On figure 1 we have marked the position of non-cleaved MASP-1 (MASP-1 zymogen) and the position of the protease domain, i.e. the B-chain (separated from the A chain since the SDS-PAGE was performed in reducing conditions), as well as a band representing a further degradation product, i.e. $\mathrm{B}^{\prime}$ chain. On the figure we have also marked the covalent complex that is formed between activated MASP-1 and C1-inhibitor. It can be seen on figure 1 that gradually more and more MASP-1 is activated during the dialysis and concentration steps.

We also determined the concentration of the different effector molecules in the purified and concentrated samples, and compared these values to the average concentrations measured in the starting sera (Table 1). The purification resulted in an almost 50-fold enrichment of MASP-1 as well as of MAp44 (48-fold and 49-fold, respectively) in the samples, whereas in the case of MASP-3 we could only detect a 15-fold enrichment. MASP1, MAp44 and MASP-3 are three splice variants of the MASP1 gene sharing the same Nterminal four domains (CUB1-EGF-CUB2-CCP1). Since the first three N-terminal domains (CUB1-EGF-CUB2) are responsible for the binding to MBL, we would have expected similar enrichment. We also see an elevation in the concentration of MASP-2 (21-fold), and MAp19 shows a 10-fold enrichment compared to the reference value.

\subsection{MBL-MASPs complexes trigger $\mathrm{Ca}^{2+}$-signaling in endothelial cells}

After purification of the MBL-MASPs complexes from human plasma we applied them to HUVECs and measured the $\mathrm{Ca}^{2+}$ signals by fluorescent microscopy. MBL-MASPs complexes containing $2 \mu \mathrm{M}$ MASP-1 triggered intracellular $\mathrm{Ca}^{2+}$ increase in endothelial cells 
in a similar way to histamine $(50 \mu \mathrm{M})$, which was used as a positive control (Fig. 2A). The kinetics of the MBL-MASPs-induced intracellular $\mathrm{Ca}^{2+}$ release is similar to what we have measured previously using recombinant MASP-1 catalytic fragment (rMASP-1cf) containing the serine protease domain and the two complement control protein modules (Megyeri et al., 2009). The MBL-MASPs-induced $\mathrm{Ca}^{2+}$ signal was dose dependent correlating with the MASP-1 content of the MBL-MASPs complexes (Fig. 2B). rMBL in the same concentration as found in the purified MBL-MASPs complexes $(32 \mu \mathrm{M})$ did not induce $\mathrm{Ca}^{2+}$ signals (Fig. 3). If we pre-incubated the MBL-MASPs complexes with $\mathrm{C} 1$ inhibitor (C1-inh) in a 1:1 molar ratio the effect was inhibited (Fig. 3).

\subsection{Proteolytically inactive forms MASP-1 catalytic fragments do not activate endothelial cells}

MASP-1 circulates in zymogen (proenzyme) form in the blood and it autoactivates only when the recognition molecule of the complex binds to target surface. We wanted to investigate whether MASP-1, and consequently the lectin pathway of complement, needs to be activated for triggering pro-inflammatory signals on endothelial cells. Previously, we created a stable proenzyme rMASP-1cf (R448Q) molecule by mutating the activation loop arginine to glutamine (Megyeri et al., 2013). This mutant cannot be cleaved by trypsin-like serine proteases and retains its one-chain proenzyme form. We added the zymogen rMASP1cf R448Q mutant to the cells and measured the $\mathrm{Ca}^{2+}$ signaling by fluorescent microscopy. The zymogen R448Q mutant did not trigger $\mathrm{Ca}^{2+}$ signals on HUVECs even at high concentration $(2 \mu \mathrm{M})$ (Fig. 4A,B), whereas the wild type rMASP-1cf as well as the MBLMASPs complexes activated the cells. This indicates that MASP-1 needs to be in active form to be able to induce pro-inflammatory signaling on endothelial cells.

As we have shown here using the MBL-MASPs complex and previously using the rMASP-1cf catalytic fragment (Megyeri et al., 2009), the effect of MASP-1 could be inhibited by pre-incubation with equimolar amount of C1-inh (Fig. 3). This suggested, but did not incontrovertibly prove that the proteolytic activity of MASP-1 is responsible for PAR4 cleavage on endothelial cell surface. C1-inh forms a covalent complex with MASP-1 (Davis et al., 2008; Paréj et al., 2013), possibly distorting its structure and preventing the access of MASP-1 to their receptors. In order to unambiguously prove that the proteolytic activity of MASP-1 is responsible for cell activation, we created a proteolytically inactive form of MASP-1 catalytic fragment, which has the conformation of the active protease. We mutated the catalytic serine residue to alanine (S646A) as previously described (Megyeri et al., 2013) 
and cleaved the $\mathrm{R}^{448}$-Ile ${ }^{449}$ activation bond by MASP-2. Thus, we produced a proteolytically inactive two-chain form of rMASP-1cf. After administration of the inactive rMASP-1cf (S646A) to HUVECs we could not detect any $\mathrm{Ca}^{2+}$-signals by fluorescent microscopy (Fig. 4A,B).

\subsection{The N-terminal region of MASP-1 and MASP-2 do not activate endothelial cells}

The MBL-MASPs complexes, besides the full-length serine proteases, contain the non-catalytic splice products of the MASP1 and MASP2 genes, MAp44 and MAp19, respectively. These molecules consist of the N-terminal interacting domains of the MASPs. In order to prove that the N-terminal non-catalytic region of MASP-1 and MASP-2 do not activate endothelial cells, we incubated HUVECs with recombinant fragments containing the three N-terminal domains (CUB1-EGF-CUB2) of the proteases. These proteins showed no detectable effect on the cells (Fig. 3), even at high concentration, corroborating that the proteolytic activity of MASP-1 is the key factor for cell activation, and other domains of the MASPs do not interact with receptors. These experiments also very strongly suggest that the two non-catalytic MBL-associated proteins, MAp19 and MAp44 do not contribute to the direct cell-activating ability of the complexes.

\subsection{MASP-3 does not activate endothelial cells}

Besides MASP-1 the MBL-MASPs complexes contain two other proteases: MASP-2 and MASP-3. We have previously shown that MASP-2 do not activate endothelial cells (Megyeri et al., 2009). In this study we also wanted to examine whether the proteolytic activity of MASP-3, the second most abundant protease of the lectin pathway, contributes to the pro-inflammatory effect. We produced the recombinant catalytic fragment (CCP1-CCP2SP) of MASP-3 (Megyeri et al., 2013). MASP-3, unlike MASP-1 and MASP-2, does not autoactivate but it can be activated by MASP-1 cleavage. We cleaved the rMASP-3cf by rMASP-1cf and carefully removed the traces of rMASP- $1 \mathrm{cf}$ form the active rMASP-3cf sample. Endothelial cells were treated with activated rMASP-3cf and the $\mathrm{Ca}^{2+}$ response was assessed. rMASP-3cf did not activate the HUVECs even at high concentration implicating that MASP-1 is the exclusive activator of the endothelial cells among the components of the MBL-MASPs complexes (Fig 3).

\section{Discussion}


The lectin pathway of the complement system is an important effector arm of the innate immunity. After the pattern recognition molecules bind to the activator structures, the associated serine proteases become activated and initiate the complement cascade resulting in several effector functions. One of the most important consequences of complement activation is the initiation of inflammation. In this work we have shown that MBL-MASPs complexes prepared from human plasma are able to directly activate endothelial cells. This is in good agreement with our previous results demonstrating that the catalytic fragment of MASP-1, containing the serine protease domain and the two complement control protein modules, cleaves protease activated receptor (PAR) 4 on the surface of endothelial cells and elicits $\mathrm{Ca}^{2+}$ response. The $\mathrm{Ca}^{2+}$ signal in the endothelial cells was proportional to the MASP-1 content of the MBL-MASPs complexes suggesting that MASP-1 is the exclusive activator of the endothelial cells in this case. Indeed, we demonstrated that the other components of the complexes: MBL, MASP-2, MASP-3, and the N-terminal domains of the MASPs are unable to elicit any response in HUVECs by themselves. These results strengthen the view that the catalytic fragment of MASP-1 (rMASP-1cf) is enzymatically equivalent to the full-length molecule; the CUB1-EGF-CUB2 region does not influence the substrate specificity. The Nterminal region, however, anchors the protease to the collagen-like stalks of the pattern recognition molecule, which might affect the accessibility of the serine protease domain to the potential substrates. This might especially concern the cell-surface substrates, such as receptors, where the accessibility is originally restricted. As our results demonstrate the MBLbound MASP-1 readily cleaves its substrate, the pattern recognition molecule does not restrict its proteolytic activity. On the contrary, the pattern recognition molecule can fix the protease near to the substrate ensuring an efficient local effect. In this respect it is important to note that MBL was shown to bind to HUVECs and this binding was increased upon LPS treatment (Oroszlán et al., 2007), however, in accordance with our present results, binding of uncomplexed (empty) MBL did not stimulate the endothelial cells. It seems plausible that in certain pathophysiological situation (e.g. bacterial infection, ischemia-reperfusion) endothelial cells express ligands for the pattern recognition molecules, which results in binding of the complexes, activation of MASP-1 and cleavage of cell-surface receptors. This proinflammatory mechanism could be especially important, since MASP-1 directly activate the cells at the very beginning of the complement cascade preceding the generation of the anaphylatoxins.

Recently it has been shown that MASP-1 also activates hepatic stellate cells (Saeed et al., 2013). Although the mechanism is not clarified yet, it seems likely that MASP-1 cleaves 
protease activated receptor on the hepatic cells. This phenomenon shows that the cellactivating ability of MASP-1 is not confined to endothelial cells.

Another recent paper reports that MBL induces cell death of proximal tubular epithelial cells (PTEC) during renal ischemia/reperfusion injury (IRI) without complement activation; i.e. no $\mathrm{C} 3$ deposition or C5b-9 complex formation was necessary for the cytotoxic effect (van der Pol et al., 2012). Although it was shown that MBL itself mediates cell death in PTEC cell culture, we cannot rule out the contribution of MASP-1 in the in vivo situation. During IRI, MBL in the serum specifically binds to the stressed cells, which is followed by the activation of associated MASP-1. The proteolytic action of MASP-1 on the PTE cells might contribute to the toxic effect immediately, preceding C3 and C5 convertase formation. This hypothesis might be checked in the future using MASP-1 specific inhibitors (Heja et al., 2012a).

The effect of the MBL-MASPs complexes and that of rMASP-1cf can be abolished by C1-inh treatment. C1-inh is a serpin (serine protease inhibitor) that makes a covalent complex with the protease molecule, distorting its structure and preventing cleavage of further substrate molecules (Beinrohr et al., 2007). We wanted to unambiguously exclude the possibility that the C1-inh mediated inhibition of the cell activating ability of MASP-1 is due to steric reasons; i.e. the complex formation with $\mathrm{C} 1$-inh simply prevents the binding the protease to the receptor and the lack of proteolytic activity plays no role in the inhibition. Neither the stable zymogen one-chain mutant form of rMASP-1cf (R448Q) nor the activation loop cleaved but proteolytically inactive mutant (S646A) was able to exert any effect on HUVECs. These results prove that the proteolytic activity of MASP-1 is essential for endothelial cell activation and the simple binding of MASP-1 to the receptor is not sufficient to provoke any response.

The ineffectiveness of zymogen (R448Q) mutant may also confirm that MASP-1 induced endothelial cell activation has an in vivo role, as in the absence of pathogen/danger associated molecular pattern (PAMP/DAMP), MASP-1 exists in a zymogen form and thus is incapable of activating endothelial cells. However, upon emergence of PAMPs/DAMPs, MBL and ficolins recognize these patterns leading to a rapid cleavage and activation of MASP-1, and this reaction is controlled by C1-inh. Anaphylatoxins are produced later in the complement cascade, and their formation is regulated by a large set of complement inhibitors beside $\mathrm{C} 1$-inh, such as C4BP, factor $\mathrm{H}$, factor I, DAF etc., therefore, MASP-1 may be effective even when the production of anaphylatoxins are blocked. Moreover, MASP-1 and anaphylatoxins may have synergistic effects, which is out of the scope of this study. 
During this study we improved the yield of MBL-MASPs complexes when purifying from human serum. The original protocol yields only the native MBL-MASPs complexes, which contain only a fraction of the total MASPs content of the serum. Most of the MASPs and MAps are associated with the ficolins whose total concentration $(781 \mathrm{nM})$ far exceeds that of MBL (48 nM) (Thiel et al. 2012). We supplemented the serum with $400 \mathrm{nM}$ recombinant MBL which dramatically increased the yield of the associated proteins. Hence it is obvious that the MASPs and MAps can migrate between the recognition molecules and the system is in dynamic equilibrium. This equilibrium can be shifted by adding empty (uncomplexed) recognition molecules to the serum. It is interesting that although complete dissociation of the MBL-MASPs complexes can be achieved only in the presence of EDTA and high ionic strength (Thiel et al., 2000), the associated proteases can readily migrate between the recognition molecules in the normal human plasma. It may also be possible that when an empty pattern recognition molecule binds to the activator surface it can recruit MASPs form the serum to generate the initiation complex. Another alternative would be that in serum there is a surplus of MASPs that are not bound to pattern recognition molecules but may be bound if we add a much higher concentration of MBL.

In conclusion, we showed that MBL-MASPs complexes are able to activate endothelial cells via the proteolytic activity of MASP-1. Our results strengthen the view that MASP-1 plays a central role in the early innate immune response. After autoactivation it initiates the lectin pathway by activating MASP-2 and MASP-3, and it also has proinflammatory activity by cleaving other substrates, such as protease activated receptor, high molecular weight kininogen (Dobó et al., 2011) and fibrinogen (Gulla et al., 2009; Hess et al., 2012). In this way MASP-1 initiates a multi-faceted response against the possibly dangerous particles recognized by the pattern recognition molecules. 
Acknowledgements: This work was supported by the Hungarian Scientific Research Fund (OTKA) grants NK100834, NK 100769, K 108642, K 100684 (LC) and the EMBO ASTF 498-2011 grant awarded to M.M. LC was granted by the „Bolyai János ${ }^{\text {ee }}$ Research Fellowship (BO/00218/10/8). 


\section{References}

Ambrus, G., Gál, P., Kojima, M., Szilágyi, K., Balczer, J., Antal, J., Gráf, L., Laich, A., Moffatt, B.E., Schwaeble, W., Sim, R.B., Závodszky, P., 2003. Natural substrates and inhibitors of mannan-binding lectin-associated serine protease-1 and -2: a study on recombinant catalytic fragments. J. Immunol. 170, 1374-1382.

Beinrohr, L., Harmat, V., Dobó, J., Lörincz, Z., Gál, P., Závodszky, P., 2007. C1 inhibitor serpin domain structure reveals the likely mechanism of heparin potentiation and conformational disease. J. Biol. Chem. 282, 21100-21109.

Dahl, M.R., Thiel, S., Matsushita, M., Fujita, T., Willis, A.C., Christensen, T., Vorup-Jensen, T., Jensenius, J.C., 2001. MASP-3 and its association with distinct complexes of the mannan-binding lectin complement activation pathway. Immunity 15, 127-135.

Davis, A.E., Mejia, P., Lu, F., 2008. Biological activities of $\mathrm{C} 1$ inhibitor. Molecular Immunology 45, 4057-4063.

Degn, S.E., Hansen, A.G., Steffensen, R., Jacobsen, C., Jensenius, J.C., Thiel, S., 2009. MAp44, a Human Protein Associated with Pattern Recognition Molecules of the Complement System and Regulating the Lectin Pathway of Complement Activation. J. Immunol. 183, 7371-7378.

Degn, S.E., Jensen, L., Gál, P., Dobó, J., Holmvad, S.H., Jensenius, J.C., Thiel, S., 2010. Biological variations of MASP-3 and MAp44, two splice products of the MASP1 gene involved in regulation of the complement system. Journal of Immunological Methods 361, 37-50.

Degn, S.E., Jensen, L., Hansen, A.G., Duman, D., Tekin, M., Jensenius, J.C., Thiel, S., 2012. Mannan-Binding Lectin-Associated Serine Protease (MASP)-1 Is Crucial for Lectin Pathway Activation in Human Serum, whereas neither MASP-1 nor MASP-3 Is Required for Alternative Pathway Function. J. Immunol. 189, 3957-3969.

Degn SE, Thiel S, Nielsen O, Hansen AG, Steffensen R, Jensenius JC. 2011. MAp19, the alternative splice product of the MASP2 gene. J Immunol Methods. 373, 89-101.

Dobó, J., Harmat, V., Sebestyén, E., Beinrohr, L., Závodszky, P., Gál, P., 2008. Purification, crystallization and preliminary X-ray analysis of human mannose-binding lectinassociated serine protease-1 (MASP-1) catalytic region. Acta Crystallogr. Sect. F Struct. Biol. Cryst. Commun. 64, 781-784.

Dobó, J., Harmat, V., Beinrohr, L., Sebestyén, E., Závodszky, P. and Gál, P. (2009) MASP-1, a Promiscuous Complement Protease: Structure of Its Catalytic Region Reveals the Basis of Its Broad Specificity. J. Immunol. 183, 1207-1214

Dobó, J., Major, B., Kékesi, K.A., Szabó, I., Megyeri, M., Hajela, K., Juhász, G., Závodszky, P., Gál, P., 2011. Cleavage of kininogen and subsequent bradykinin release by the complement component: mannose-binding lectin-associated serine protease (MASP)- 1 . PLoS ONE 6, e20036.

Gál, P., Harmat, V., Kocsis, A., Bián, T., Barna, L., Ambrus, G., Végh, B., Balczer, J., Sim, R.B., Náray-Szabó, G., Závodszky, P., 2005. A true autoactivating enzyme. Structural insight into mannose-binding lectin-associated serine protease-2 activations. J. Biol. Chem. 280, 33435-33444.

Gulla, K.C., Gupta, K., Krarup, A., Gál, P., Schwaeble, W.J., Sim, R.B., O’Connor, C.D., Hajela, K., 2009. Activation of mannan-binding lectin-associated serine proteases leads to generation of a fibrin clot. Immunology 129, 482-495.

Hansen S., Selman L., Palaniyar N., Ziegler K., Brandt J., Kliem A., Jonasson M., Skjoedt M. O., Nielsen O., Hartshorn K., Jorgensen T. J., Skjodt K. and Holmskov U. (2010) Collectin 11 (CL-11, CL-K1) is a MASP-1/3-associated plasma collectin with microbial- 
binding activity. J Immunol 185, 6096-104.

Heja, D., Harmat, V., Fodor, K., Wilmanns, M., Dobo, J., Kekesi, K.A., Zavodszky, P., Gal, P., Pal, G., 2012a. Monospecific Inhibitors Show That Both Mannan-binding Lectinassociated Serine Protease-1 (MASP-1) and -2 Are Essential for Lectin Pathway Activation and Reveal Structural Plasticity of MASP-2. Journal of Biological Chemistry 287, 20290-20300.

Heja, D., Kocsis, A., Dobó, J., Szilágyi, K., Szász, R., Závodszky, P., Pál, G., Gál, P., 2012b. Revised mechanism of complement lectin-pathway activation revealing the role of serine protease MASP-1 as the exclusive activator of MASP-2. Proceedings of the National Academy of Sciences 109, 10498-10503.

Hess, K., Ajjan, R., Phoenix, F., Dobó, J., Gál, P., Schroeder, V., 2012. Effects of MASP-1 of the Complement System on Activation of Coagulation Factors and Plasma Clot Formation. PLoS ONE 7, e35690.

Jensenius, J.C., Jensen, P.H., McGuire, K., Larsen, J.L., Thiel, S., 2003. Recombinant mannan-binding lectin (MBL) for therapy. Biochem Soc Trans. 31, 763-767.

Kidmose, R.T., Laursen, N.S., Dobó, J., Kjaer, T.R., Sirotkina, S., Yatime, L., Sottrup-Jensen, L., Thiel, S., Gál, P., Andersen, G.R., 2012. Structural basis for activation of the complement system by component C4 cleavage. Proceedings of the National Academy of Sciences 109, 15425-15430.

Kocsis, A., Kékesi, K.A., Szász, R., Végh, B.M., Balczer, J., Dobó, J., Závodszky, P., Gál, P., Pál, G., 2010. Selective inhibition of the lectin pathway of complement with phage display selected peptides against mannose-binding lectin-associated serine protease (MASP)-1 and -2: significant contribution of MASP-1 to lectin pathway activation. The Journal of Immunology 185, 4169-4178.

Matsushita, M., Endo, Y., Fujita, T., 2000a. Cutting edge: complement-activating complex of ficolin and mannose-binding lectin-associated serine protease. J. Immunol. 164, 22812284.

Matsushita, M., Fujita, T., 1992. Activation of the classical complement pathway by mannose-binding protein in association with a novel $\mathrm{C} 1$ s-like serine protease. J. Exp. Med. 176, 1497-1502.

Matsushita, M., Thiel, S., Jensenius, J.C., Terai, I., Fujita, T., 2000b. Proteolytic activities of two types of mannose-binding lectin-associated serine protease. J. Immunol. 165, 26372642.

Megyeri, M., Harmat, V., Major, B., Vegh, A., Balczer, J., Heja, D., Szilagyi, K., Datz, D., Pal, G., Zavodszky, P., Gal, P., Dobo, J., 2013. Quantitative Characterization of the Activation Steps of Mannan-binding Lectin (MBL)-associated Serine Proteases (MASPs) Points to the Central Role of MASP-1 in the Initiation of the Complement Lectin Pathway. Journal of Biological Chemistry 288, 8922-8934.

Megyeri, M., Makó, V., Beinrohr, L., Doleschall, Z., Prohászka, Z., Cervenak, L., Závodszky, P., Gál, P., 2009. Complement protease MASP-1 activates human endothelial cells: PAR4 activation is a link between complement and endothelial function. J. Immunol. 183, 3409-3416.

Møller-Kristensen, M., Jensenius, J.C., Jensen, L., Thielens, N., Rossi, V., Arlaud, G., Thiel, S., 2003. Levels of mannan-binding lectin-associated serine protease- 2 in healthy individuals. Journal of Immunological Methods 282, 159-167.

Oroszlán, M., Daha, M.R., Cervenak, L., Prohászka, Z., Füst, G., Roos, A., 2007. MBL and C1q compete for interaction with human endothelial cells. Molecular Immunology 44, 1150-1158.

Paréj, K., Dobó, J., Závodszky, P., Gál, P., 2013. The control of the complement lectin 
pathway activation revisited: both $\mathrm{C} 1$-inhibitor and antithrombin are likely physiological inhibitors, while a2-macroglobulin is not. Molecular Immunology 54, 415-422.

Paréj K, Hermann A, Donáth N, Závodszky P, Gál P, Dobó J. (2014) Dissociation and reassociation studies on the interaction domains of mannan-binding lectin (MBL)associated serine proteases, MASP-1 and MASP-2, provide evidence for heterodimer formation. Mol Immunol. 59, 1-9.

Ricklin, D., Hajishengallis, G., Yang, K., Lambris, J.D., 2010. Complement: a key system for immune surveillance and homeostasis. Nat. Immunol. 11, 785-797.

Saeed, A., Baloch, K., Brown, R.J.P., Wallis, R., Chen, L., Dexter, L., McClure, C.P., Shakesheff, K., Thomson, B.J., 2013. Mannan binding lectin-associated serine protease 1 is induced by hepatitis $\mathrm{C}$ virus infection and activates human hepatic stellate cells. Clin Exp Immunol n/a-n/a.

Skjoedt, M.O., Hummelshoj, T., Palarasah, Y., Honore, C., Koch, C., Skjodt, K., Garred, P., 2010. A Novel Mannose-binding Lectin/Ficolin-associated Protein Is Highly Expressed in Heart and Skeletal Muscle Tissues and Inhibits Complement Activation. Journal of Biological Chemistry 285, 8234-8243.

Stover, C.M., Thiel, S., Thelen, M., Lynch, N.J., Vorup-Jensen, T., Jensenius, J.C., Schwaeble, W.J., 1999. Two constituents of the initiation complex of the mannan-binding lectin activation pathway of complement are encoded by a single structural gene. J. Immunol. 162, 3481-3490.

Takahashi, M., Endo, Y., Fujita, T., Matsushita, M., 1999. A truncated form of mannosebinding lectin-associated serine protease (MASP)-2 expressed by alternative polyadenylation is a component of the lectin complement pathway. International Immunology 11, 859-863.

Thiel, S., 2007. Complement activating soluble pattern recognition molecules with collagenlike regions, mannan-binding lectin, ficolins and associated proteins. Molecular Immunology 44, 3875-3888.

Thiel, S., Jensen, L., Degn, S.E., Nielsen, H.J., Gal, P., Dobo, J., Jensenius, J.C., 2012. Mannan-binding lectin (MBL)-associated serine protease-1 (MASP-1), a serine protease associated with humoral pattern-recognition molecules: normal and acute-phase levels in serum and stoichiometry of lectin pathway components. Clin Exp Immunol 169, 38-48.

Thiel, S., Møller-Kristensen, M., Jensen, L., Jensenius, J.C., 2002. Assays for the functional activity of the mannan-binding lectin pathway of complement activation. Immunobiology 205, 446-454.

Thiel, S., Petersen, S.V., Vorup-Jensen, T., Matsushita, M., Fujita, T., Stover, C.M., Schwaeble, W.J., Jensenius, J.C., 2000. Interaction of C1q and mannan-binding lectin (MBL) with C1r, C1s, MBL-associated serine proteases 1 and 2, and the MBL-associated protein MAp19. J. Immunol. 165, 878-887.

Thiel, S., Vorup-Jensen, T., Stover, C.M., Schwaeble, W., Laursen, S.B., Poulsen, K., Willis, A.C., Eggleton, P., Hansen, S., Holmskov, U., Reid, K.B., Jensenius, J.C., 1997. A second serine protease associated with mannan-binding lectin that activates complement. Nature 386, 506-510.

van der Pol, P., Schlagwein, N., van Gijlswijk, D.J., Berger, S.P., Roos, A., Bajema, I.M., de Boer, H.C., de Fijter, J.W., Stahl, G.L., Daha, M.R., van Kooten. C., 2012. Mannan-binding lectin mediates renal ischemia/reperfusion injury independent of complement activation. Am J Transplant. 12, 877-887. 


\section{Figure legends}

Figure 1. MASP-1 autoactivates in the MBL-MASPs complexes during purification. MBL-MASPs complexes were purified on mannan-Sepharose from serum to which recombinant MBL had been added. The figure shows western blot analysis of reduced samples from the last steps of purification developed with Rabbit 64 antiserum recognizing MASP-1 B and B' chains under reducing conditions. The MASP-1 zymogen disappears from the sample during dialysis and concentration and is found in the activated (two chain) form. Lane 1 is the sample after elution from the mannan-Sepharose $4 \mathrm{~B}$ affinity column. Lane 2 is the sample after dialysis against HBSS buffer. Lane 3 is the sample after removing the precipitate formed during dialysis and Lane 4 is the concentrated stock used for experiments on endothelial cells. Molecular weight markers are shown on the right hand side and the positions of the MASP-1 related bands are indicated on the left hand side.

\section{Figure 2. MBL-MASPs complexes trigger intracellular $\mathrm{Ca}^{2+}$ - signaling in HUVECs.} Intracellular $\mathrm{Ca}^{2+}$-signaling was measured by fluorescence microscopy in every $5 \mathrm{~s}$ for $115 \mathrm{~s}$ after activation of HUVECs loaded with Fluo-4 dye. MBL-MASPs complexes, and histamine as a positive control, were added at $15 \mathrm{~s}$. A, Representative kinetic curves of calcium response in HUVEC triggered by $50 \mu \mathrm{M}$ Histamine and MBL-MASPs complexes containing $2 \mu \mathrm{M}$ MASP-1. Mean fluorescent measurements of 20 cells with SEM are plotted against time. B, Dose-dependent calcium response induced by MBL-MASPs complexes containing 0.2, 0.6 or $2 \mu \mathrm{M}$ MASP-1. Maximum green fluorescent values (mean of 20 cells) were normalized by the response to that of $50 \mu \mathrm{M}$ Histamine. Means \pm SEM of 3 independent experiments performed on HUVECs from different donors are presented. The significance compared to buffer control was calculated by one way ANOVA. ***: $\mathrm{p}<0.001$ ns: non significant.

Figure 3. The $\mathrm{Ca}^{2+}$ mobilization ability of MBL-MASPs complexes, rMASP-1cf, MBL, rMASP-3cf and the N-terminal fragments of MASPs. The $\mathrm{Ca}^{2+}$ mobilization as the percentage of signal induced by histamine is shown. MBL-MASPs complexes containing 1 $\mu \mathrm{M}$ MASP-1 were preincubated with equimolar amount of $\mathrm{C} 1$-inh before treatment of the cells. rMASP-3cf consists of the CCP1-CCP2-SP domains in the active conformation (see Materials and Methods). The N-terminal fragments of MASP-1 and -2 comprise the respective CUB1-EGF-CUB2 domains of the proteins. Maximum green fluorescent values (mean of 20 cells) in the percentage of histamine were calculated, and mean \pm SEM of 3 
independent experiments performed on HUVECs from different donors are presented. $* * *: \mathrm{p}<$ $0.001 \mathrm{~ns}$ : non significant.

Figure 4. Proteolytically inactive MASP-1 catalytic fragments do not activate endothelial cells. The intracellular calcium signal triggered by zymogen R448Q rMASP-1cf (abbreviated as RQ-mutant) and catalytically inactive S646A rMASP-1cf (abbreviated as SA-mutant) was measured. The S646A fragment was brought into active conformation as described in the text. A, Representative kinetic curves of calcium response in HUVECs triggered by $2 \mu \mathrm{M} w \mathrm{w}$, S646A or R448Q rMASP-1cf fragments. Thrombin (100 nM) was used as a positive control. Mean fluorescent measurements of 20 cells with SEM are plotted against time. B, Calcium mobilization of proteolytically inactive mutants S646A and R448Q are shown as the percentage of fluorescent signal induced by $100 \mathrm{nM}$ thrombin. Means of maximum green fluorescent values of 20 cells were calculated and, mean \pm SEM of 3 independent experiments performed on HUVECs from different donors are presented. ***: $\mathrm{p}<0.001 \mathrm{~ns}$ : non significant. 
Fig. 1.

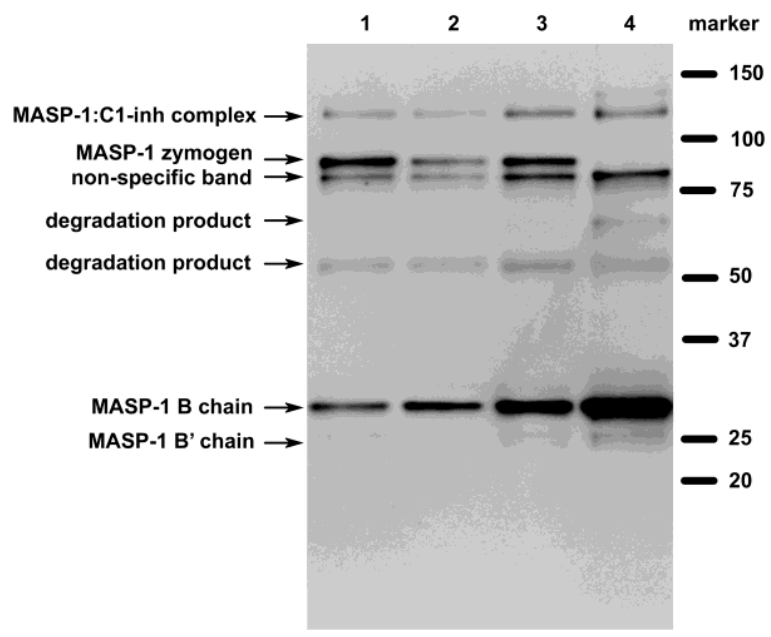


Fig. 2.
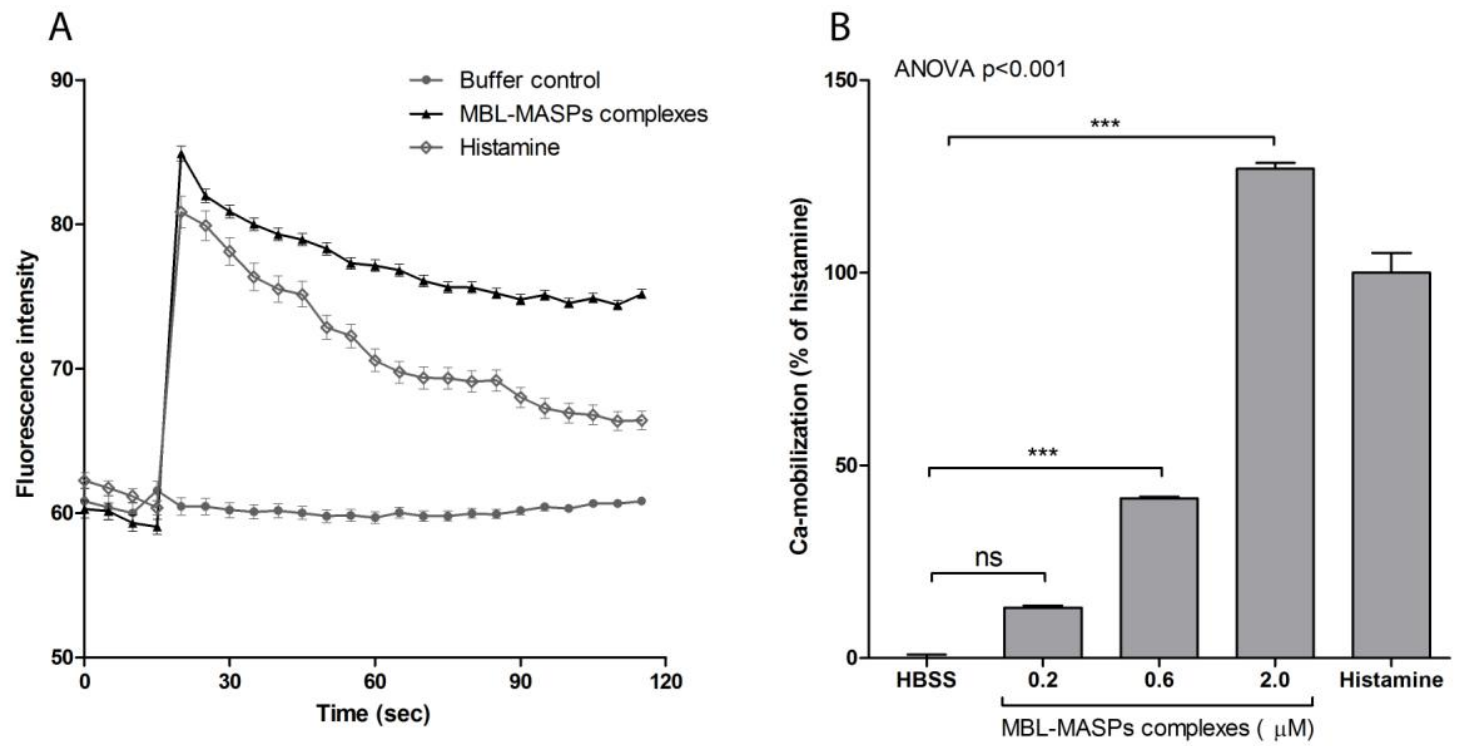
Fig. 3.

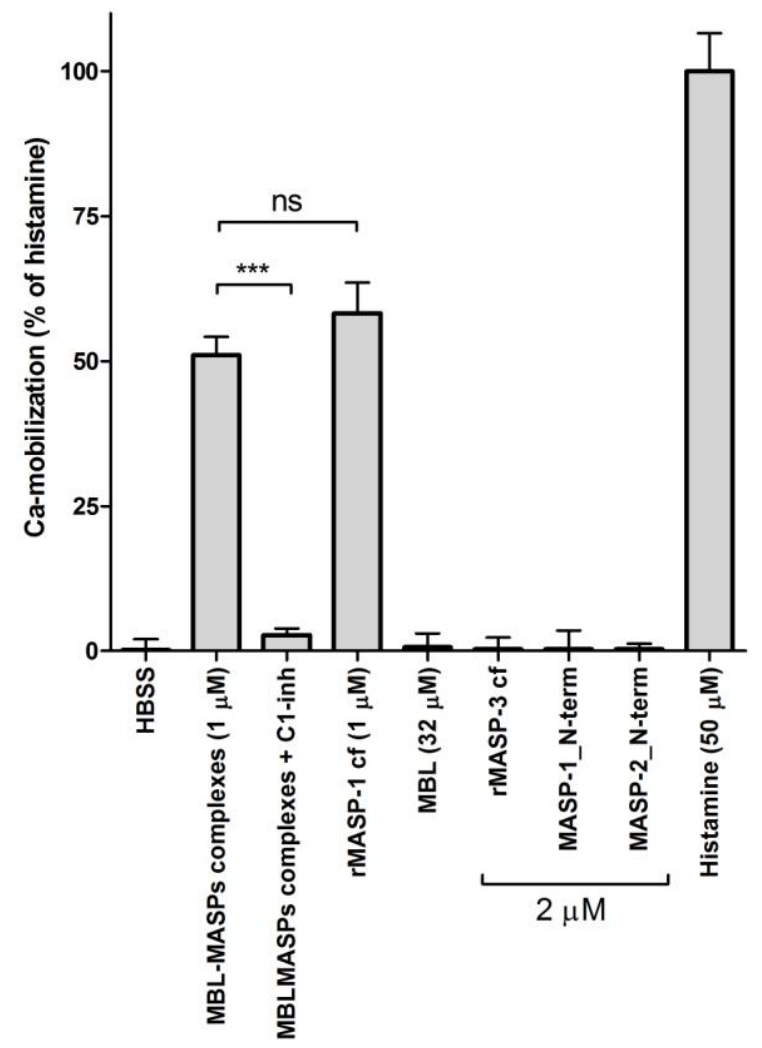


Fig. 4.
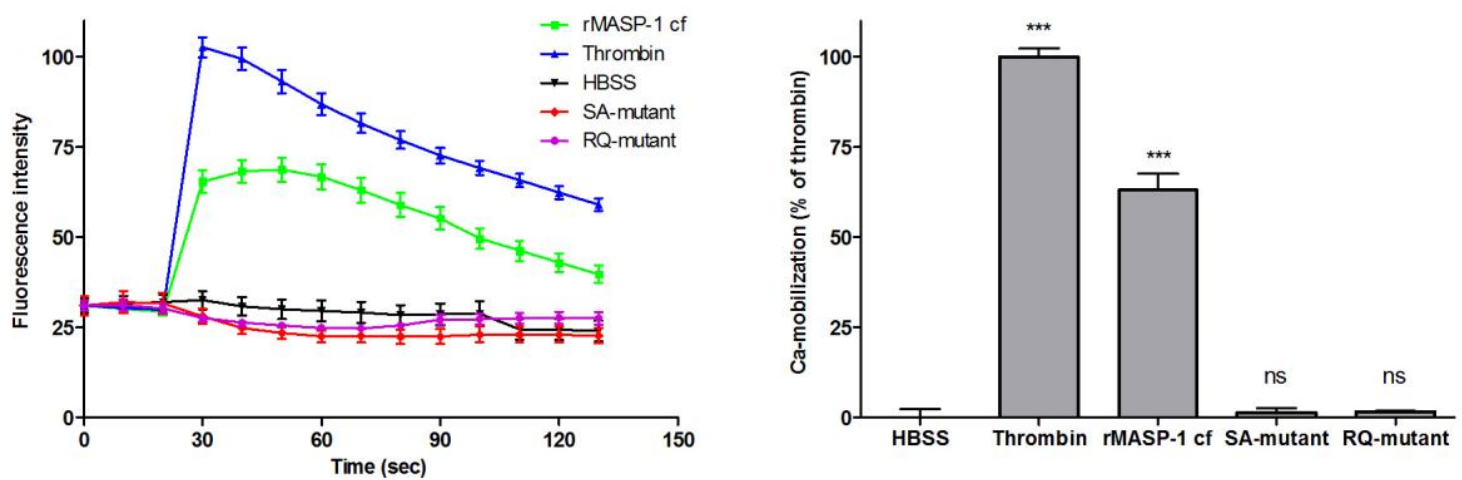
Table 1.

Table 1. Concentration of MBL-associated proteases (MASPs) and proteins (MAps) in plasma and in purified samples

\begin{tabular}{lccccc} 
& MASP-1 & MASP-3 & MAp44 & MASP-2 & MAp19 \\
\hline \hline${\text { Initial conc. }{ }^{\mathrm{a}}(\mu \mathrm{g} / \mathrm{ml})}^{9.6}$ & 4.6 & 1.4 & 0.3 & NM $^{\mathrm{b}}$ \\
Average conc. $^{\mathrm{c}}(\mu \mathrm{g} / \mathrm{ml})$ & 11.0 & 5.0 & 1.6 & 0.4 & 0.4 \\
Purified sample conc. $(\mu \mathrm{g} / \mathrm{ml})$ & 466.0 & 70.0 & 68.0 & 6.0 & 4.0 \\
\hline Purified / initial conc. ratio & 48.5 & 15.2 & 48.6 & 20.0 & $\mathrm{NA}^{\mathrm{d}}$ \\
Purified /average conc. ratio & 42.4 & 14.0 & 42.5 & 15.0 & 10.0 \\
\hline
\end{tabular}

${ }^{a}$ Measured from the starting plasma samples using TRIFMA assay.

${ }^{\mathrm{b}} \mathrm{NM}$, not measured

${ }^{c}$ Average serum concentrations determined by Thiel et al. (Thiel et al., 2012)

${ }^{\mathrm{d}} \mathrm{NA}$, not applicable 